buffers. More local patterns of area effect are further revealed, eg more mixed land use is associated with higher levels of walking in some but not all areas of the city, suggesting that the significance and magnitude of associations between built environment characteristics and walking are not the same across space.

Discussion: Application and findings of the three approaches lead to distinct yet complementary conclusions about the environmental determinants of physical activity and the scales at which they may be operating. Findings are discussed in terms of their implications for public health practice and policy, eg acquiring and inventorying data on population health as well as area characteristics for surveillance purposes, developing and implementing health promotion and prevention strategies based on extent data at the local and at different geographical levels, and evaluating policy interventions aimed at changing the local environment. The challenges of targeting actions at the local area level are underlined and examined in light of recent national (UK and Canada) and international recommendations and guidance to create environments conducive to physical activity.

\section{THE 2008 GREAT NORTH RUN AS PUBLIC HEALTH INTERVENTION: PARTICIPANT CHARACTERISTICS AND CHANGES IN PHYSICAL ACTIVITY AND BODY MASS INDEX FROM 20 WEEKS BEFORE TO 20 WEEKS AFTER THE EVENT}

J Adams, M White. Institute of Health and Society, William Leech Building, Newcastle University, Newcastle upon Tyne, UK

\section{doi:10.1136/jech.2009.096701k}

Background: Mass participation sporting events are often proposed as methods of promoting physical activity and decreasing obesity. Little research has explored who takes part in such events or if participation leads to sustained behaviour change. The Great North Run (GNR), from Newcastle upon Tyne to South Shields (13.1 miles), is the UK's largest mass participation sporting event. Objectives: To describe the characteristics of non-elite runners in the 2008 GNR, and document group-level changes in physical activity and body mass index (BMI) between 20 weeks before (time 1) and 20 week after (time 2) the event.

Design: Age, gender and postcode of residence for all 2008 GNR nonelite runners was provided by the organisers ( $n=51$ 185). Links to online surveys were sent to all non-elite runners via email at times 1 and 2. These collected information on age, gender, current physical activity levels (using the short International Physical Activity Questionnaire, IPAQ), self reported height and weight, and postcode of residence. IPAQ responses were used to categorise physical activity over the past seven days as low, moderate or high. Postcodes of residence were used to assign Index of Multiple Deprivation data to those runners living in England (91.7\% of all non-elite runners).

Results: Median age of all 51185 non-elite runners was 35.6 (IOR 28.4 to 43.7 ) years, $58.9 \%$ were male and $53.5 \%$ of those living in England lived in the least deprived $40 \%$ of areas. 3018 participants responded to the survey at time 1 and 2851 at time 2 . Respondents to both surveys were slightly older and less likely to be male than all non-elite runners but had a similar deprivation profile. Among respondents at time 1 , physical activity was categorised as high in $65.0 \%$ and moderate in 29.2\%; median BMI was 24.0 (IOR 22.0 to 26.3). At time 2, physical activity was categorised as high in $68.0 \%$ and moderate in 21.8\%. Median BMI was 24.0 (IOR 21.9 to 26.3). Conclusions: The typical GNR participant is male, mid-30s, lives in an area of low deprivation, has a "normal" BMI, and is moderately or highly active. Group-level differences in physical activity and BMI between those responding at time 1 and 2 were minimal. The repeat cross-sectional design means that individuallevel conclusions cannot be drawn. GNR participants are not those who could most benefit from increased physical activity. The GNR may provide a focus for maintaining activity and BMI, but there is little evidence that participation leads to sustained group-level improvements in either measure.

\section{HEALTHY EATING AND PHYSICAL ACTIVITY CHOICES IN SOCIO- ECONOMICALLY DISADVANTAGED WOMEN: THE ROLE OF HEALTH LITERACY}

J Edwards, C Jackson, F Cheater, J Holt, M Robinson. School of Healthcare, University of Leeds, Leeds, UK

\section{doi:10.1136/jech.2009.0967011}

Background: The Public Health White Paper recognises the need to help individuals to make informed decisions about healthy eating and physical activity. However, one in five people in the UK cannot interpret basic health information. Poor health literacy is associated with socio-economic disadvantage and compounds health inequalities. Interventions to promote informed decision-making for healthy eating and physical activity commonly ignore patient health literacy. Indeed, there is a lack of research into health literacy and lifestyle choices as well as poor understanding of how different population groups seek, interpret, critically analyse and use information to make informed lifestyle decisions. This novel study explored health literacy for healthy eating and physical activity decisions in socio-economically disadvantaged women. We focused on women because they typically have responsibility for family health. Improving health literacy in disadvantaged communities may confer greatest public health benefit.

Methods: Eighty five women living in deprived areas of three northern cities were theoretically sampled to provide a diversity of age, ethnicity and educational background. Rootman's health literacy model provided the theoretical framework for the study. Semi-structured interviews explored how women seek, understand, trust and use healthy eating and physical activity information/advice to make informed decisions. Interviews were recorded, transcribed verbatim and are currently being analysed using the Framework approach.

Findings: Emerging themes suggest that women access lifestyle information/advice through various sources including community groups, health centres, media, family and friends. Many report a lack of available information on physical activity. Understanding of the components of healthy eating (eg 5-a-day) and physical activity (eg 5 weekly bouts of 30 minutes) varies markedly across women and is challenged by confusing, mixed messages in the media and health literature. Many women find practical demonstrations, eg of what constitutes a "portion" in combination with oral delivery of information as helpful for understanding. Trustworthy sources of lifestyle information include the NHS, family and friends. Interestingly whilst women were wary of commercially-produced health information, they trusted Weight Watchers. Finally, women talked about how their use of information to make healthy choices is often constrained by issues peripheral to health literacy, eg family circumstances and finance.

Conclusion: These preliminary findings highlight the important role that health literacy plays in the adoption of healthy lifestyles. They also identify potential ways to improve the quality of support and advice provided by health, community and social care professionals.

\section{Inequalities I}

\section{ETHNICITY AND CANCER TREATMENT IN NEW ZEALAND: DO MAORI PATIENTS GET A WORSE DEAL?}

${ }^{1} S$ Hill, ${ }^{2} \mathrm{D}$ Sarfati, ${ }^{2} \mathrm{~T}$ Blakely, ${ }^{2} \mathrm{~B}$ Robson. 'University of Edinburgh, Edinburgh, UK; ${ }^{2}$ University of Otago, Dunedin, New Zealand

doi:10.1136/jech.2009.096701m

Background: Ethnic or racial disparities in cancer treatment have been documented in several countries and cancer types. We 